\title{
The Relationship Between Self-Efficacy and Mental Health in Women with Breast Cancer Referred to Health Centers in Hamadan 2016-2017
}

\author{
Nahid Mohammadi ${ }^{1}$, Hengameh Pazhoohnia ${ }^{2}$, Masoud Khodaveisi ${ }^{* 3}$, Alireza Soltanian ${ }^{4}$, Sana Niknam ${ }^{5}$ \\ 1. Instructor, Department of Comunity Health Nursing, Chronic Diseases (Home Care) Research Center, Hamadan \\ University of Medical Sciences, Hamadan, Iran \\ 2. MSc Student of Comunity Health Nursing, Department of Nursing, School of Nursing and Midwifery, Hamadan \\ University of Medical Sciences, Hamadan, Iran \\ 3. Associate Professor, Department of Comunity Health Nursing, Chronic Diseases (Home Care) Research Center, \\ Hamadan University of Medical Sciences, Hamadan, Iran \\ 4. Associate Professor, Modeling of Noncommunicable Disease Research Center, Hamadan University of Medical \\ Sciences, Hamadan, Iran \\ 5. BS of Nursing, Hamadan University of Medical Sciences, Hamadan, Iran
}

Article Info $\quad$ Abstract

$\begin{array}{ll}\text { Received: } & \text { 2017/06/12 } \\ \text { Accepted: } & \text { 2017/07/8 } \\ \text { Published Online } & \text { 2017/07/17 }\end{array}$

DOI: $10.30699 /$ sjhnmf.26.2.72

Original Article

Use your device to scan and read the article online

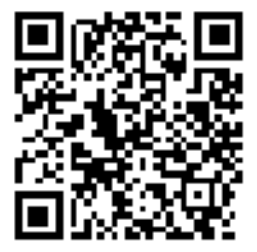

Introduction: Breast cancer is the most common malignant disease in women which compromises person's spiritual health. Given the importance of spiritual health and its effect on self-efficacy, the aim of this study was to determine the relationship between self-efficacy and mental health in women with breast cancer.

Methods: This Cross-Sectional correlative study was conducted on 196 women with breast cancer referred to hematology and intensive care unit of Shahid Beheshti Hospital, medical center and Mahdiei MRI clinic in Hamadan during 2016-2017. The study subjects were selected by simple convenience sampling method. The measurement tools were Demographic questionnaire, the Sherer self-efficacy scale and Palutziam and Ellison spiritual health which was completed by patients. Data was analyzed by SPSS18 using statistical methods including Pearson correlation coefficient, independent t-test, ANOVA and logistic regression.

Results: The score of majority of women in spiritual well-being (43/87\%) and efficacy $(41 / 83 \%)$ were in a moderate level. The mean number of spiritual health was $55.92 \pm 2.48$ and the mean number self-efficacy was $44.33 \pm 3.08$, respectively and both of them were average. . There was positive significant correlation between self-efficacy and spiritual health $(P<0 / 01 \mathrm{r}=0.04)$. There was also significant association between all demographic characteristics and spiritual health and other demographic characteristics including (education, breastfeeding frequency, type of insurance, duration of breast cancer and involved lobe) and self-efficacy $(P<0 / 05)$.

Conclusion: With regards to relationship between self-efficacy and spiritual health, it seems that in order to enhance the self-efficacy in patients with other chronic diseases such as cancer attention to spiritual health is recommended.

Keywords: Spiritual Health, Self-Efficacy, Breast Cancer

Masoud Khodaveisi, Associate Professor, Department of Comunity Health Nursing, Chronic Diseases

Copyright $($ C 2018, Sci J Hamadan Nurs Midwifery Fac. This is an open-access article distributed under the terms of the Creative Commons Attribution-noncommercial 4.0 International License which permits copy and redistribute the material just in noncommercial usages, provided the original work is properly cited.

How to Cite This Article:

Mohammadi N, Pazhoohnia H, Khodaveisi M, Soltanian A, Niknam S. The Relationship Between Self-Efficacy and Mental Health in Women with Breast Cancer Referred to Health Centers in Hamadan 20162017. Sci J Hamadan Nurs Midwifery Fac. 2018; 26 (2): 72-81 


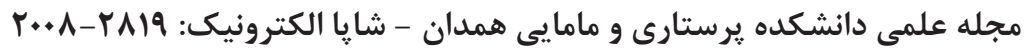

مقالهُ يزوهشى

بررسى همبستخى خود كار آمدى با سلامت معنوى زنان مبتلا به سرطان سينة مراجعه كننده به مراكز درمانى

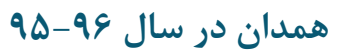

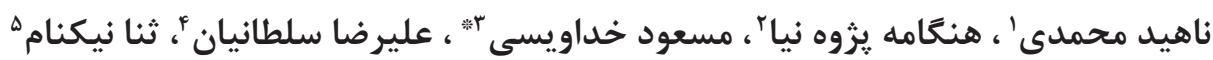

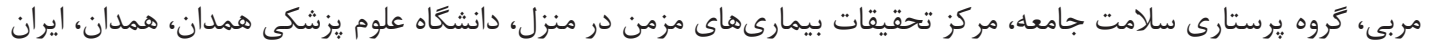

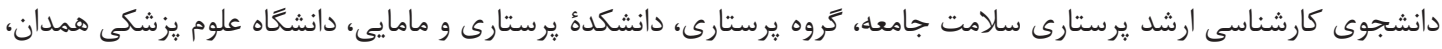
همدان، ايران

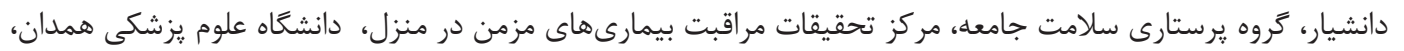
همدان، ايران

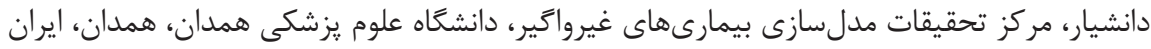

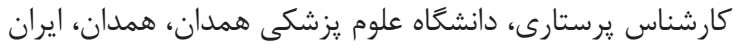
.r r .

جكيده

مقدمه: سرطان يستان شايعترين بيمارى بدخيم زنان است كه سلامت معنوى فرد را به خطر مىاندازد. با توجه

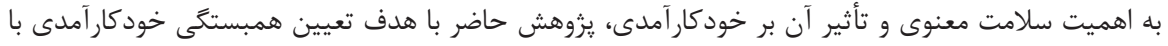

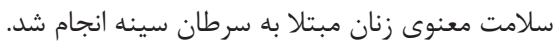

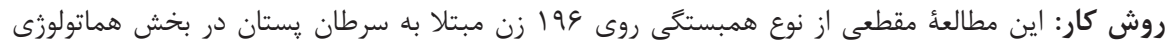

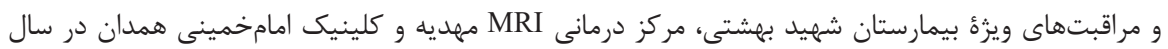

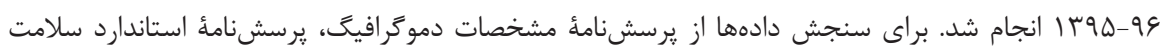
معنوى گولتزين و اليسون و خودكارآمدى عمومى شرر استفاده شد كه از سوى بيماران تكميل و در نهايت دادهانها

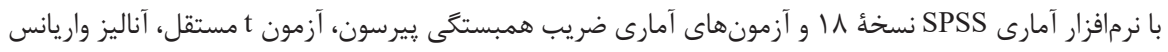
يك طرفه و ركرسيون لجستيك تجزيلورتحليل شدند.

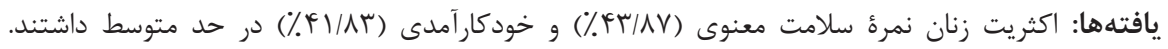
ميانخين نمره سلامت معنوى

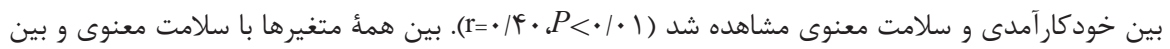

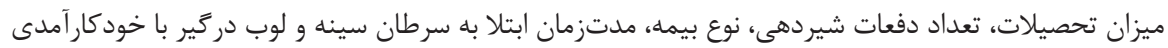

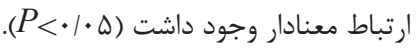

نتيجه گيرى: از آنجايى كه بين خود كار آمدى با سلامت معنوى ارتباط معنى دارى وجود داشت، لذا براى بالا بردن خودكارآمدى بيماران مبتلا به ساير بيمارىهاى مزمن از جمله سرطان، توجه به سلامت معنوى توصيه مى شىود.

وازًَان كليدى: سلامت معنوى، خود كارآمدى، سرطان پِتان

اطلاعات مقاله

تاريخ وصول: : ت : ت

تاريخ يذيرش:

انتشار آنلاين:

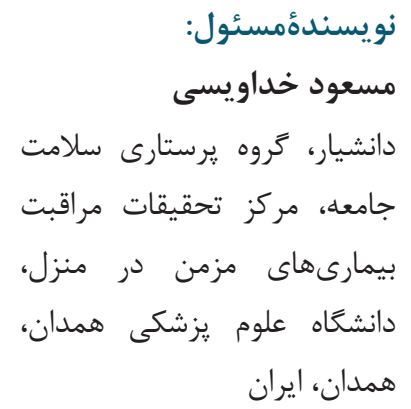

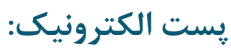

khodaveisi@umsha.ac.ir

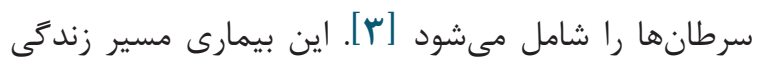

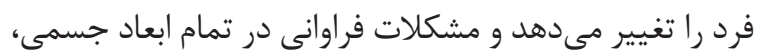

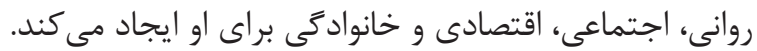

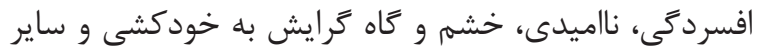

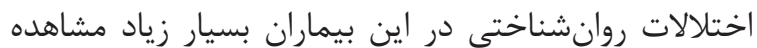

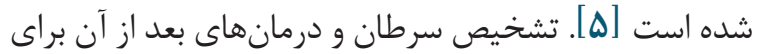

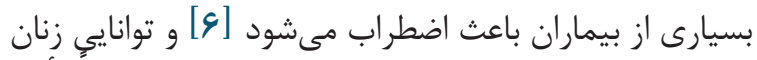

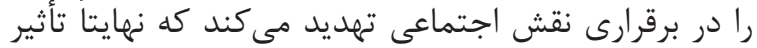

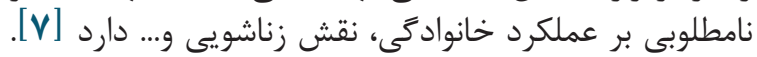

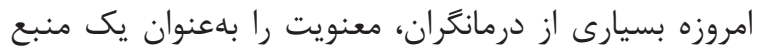

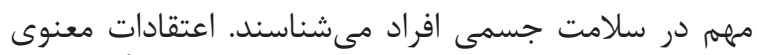

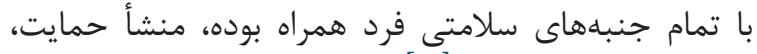

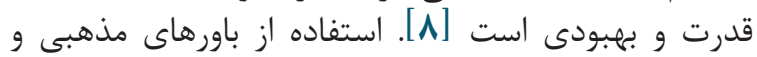

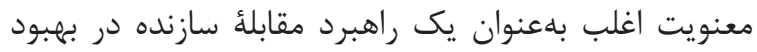

مقدمه

سرطان بهعنوان يكى از مهمترين بيمارىهاى قرن حاضر

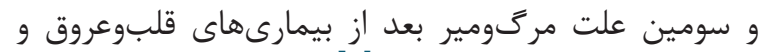

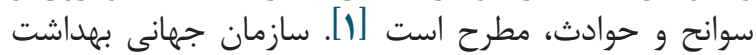

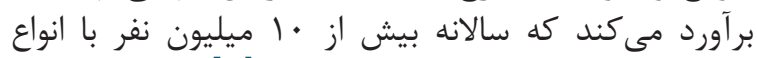

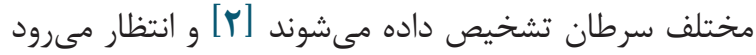

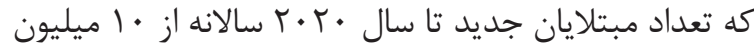

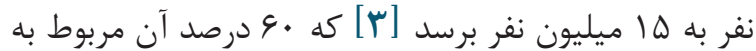

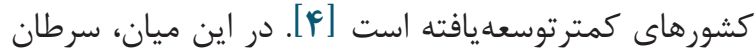

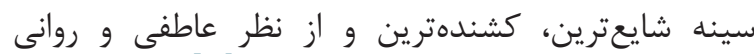

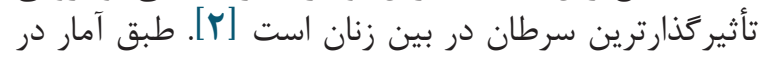

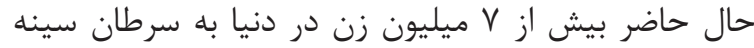

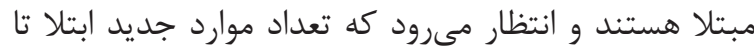

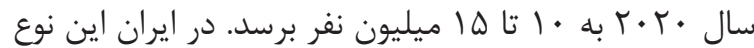

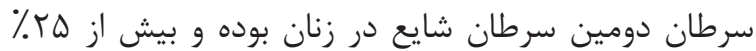


درمانى همدان انجام شد.

روش كار

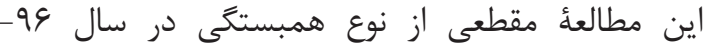

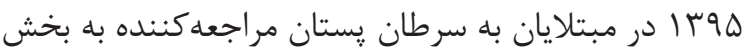

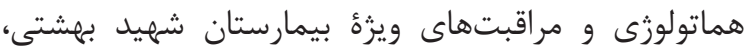

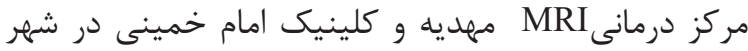

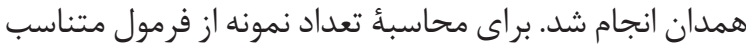

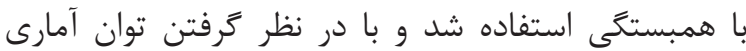

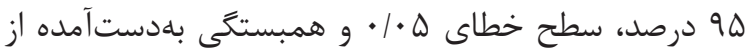

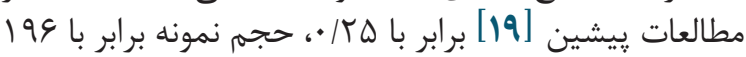
بهدست آمده است.

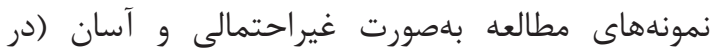

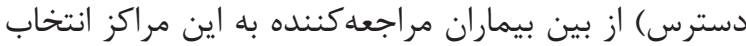

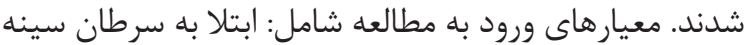

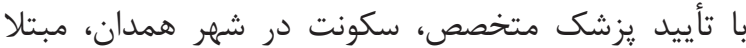

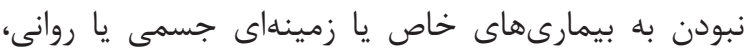

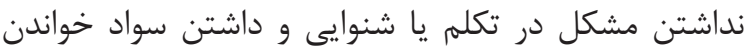

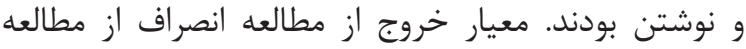

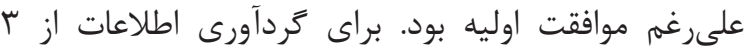

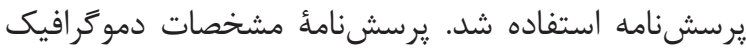

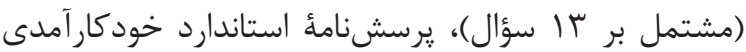

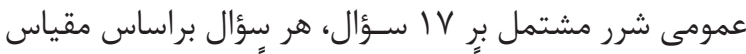

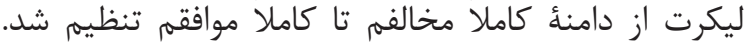

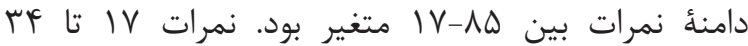

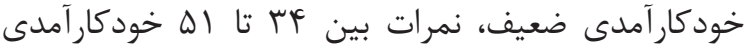

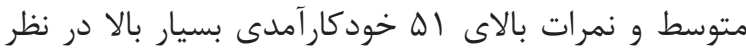

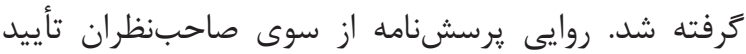

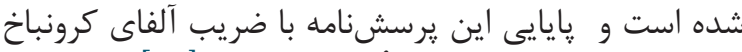

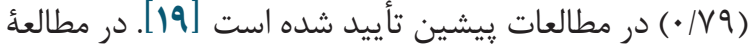

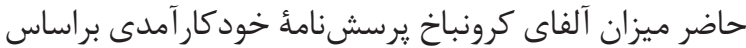

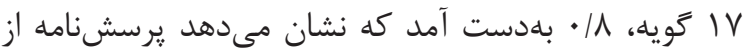

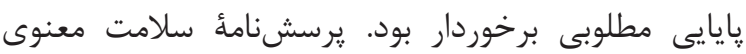

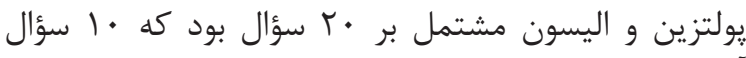

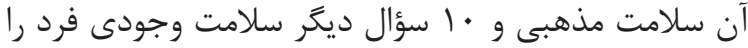

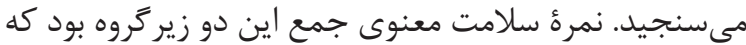

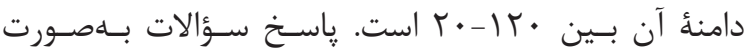

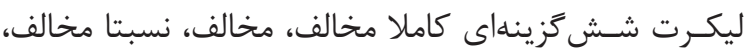

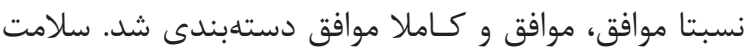

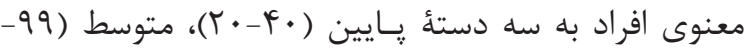

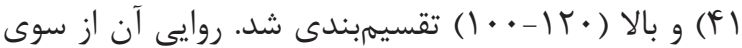

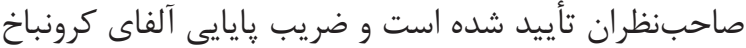

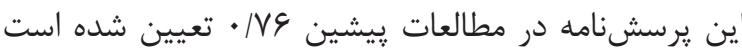

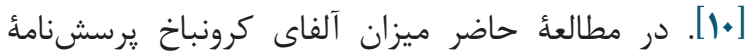

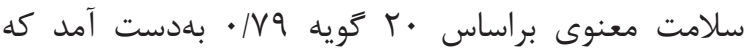

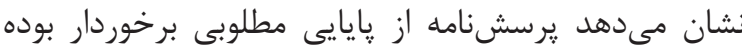

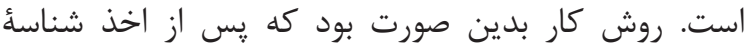

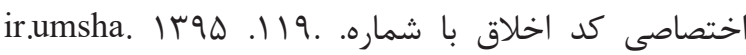
rec و مجوزهاى لازم بلهصورت مستمر طى

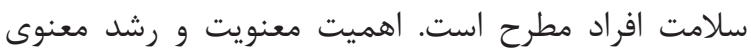

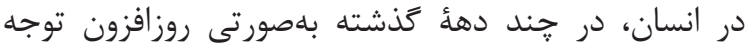

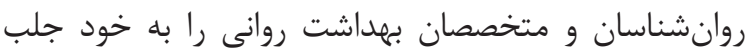
كرده است. به طورى كه سازمان بهداشت جهان جهانى در بعان تعريف

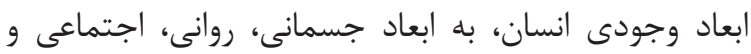

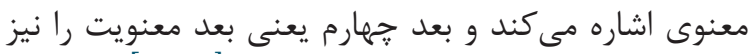

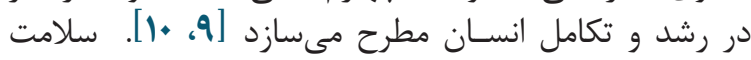

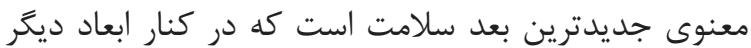

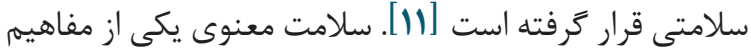

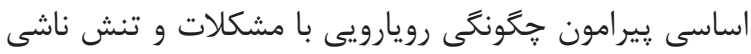

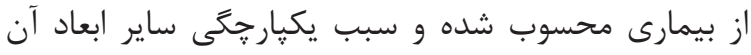

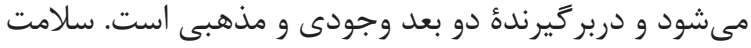

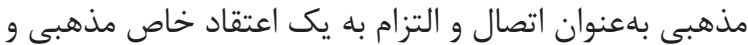

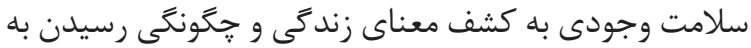

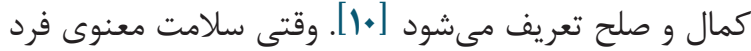

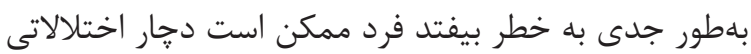

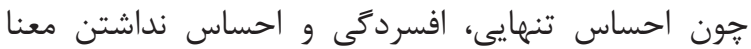

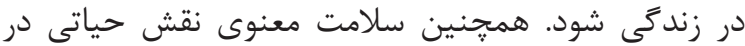

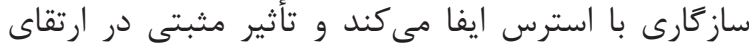

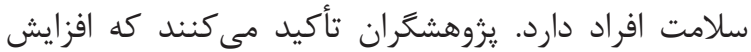

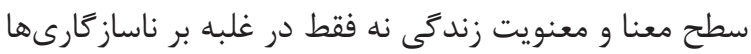

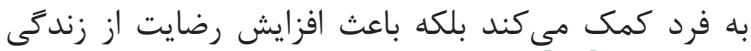

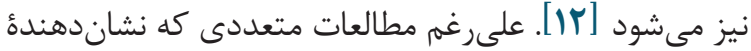

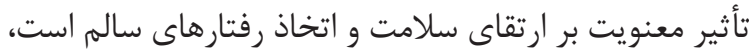

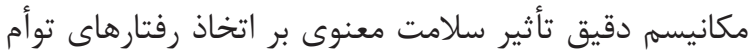

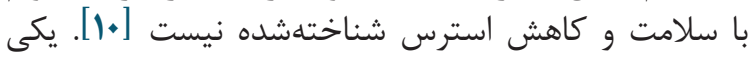

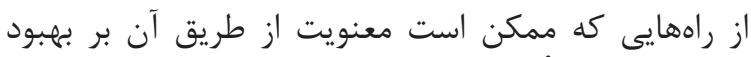

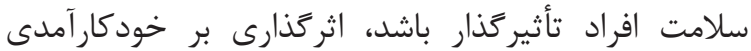

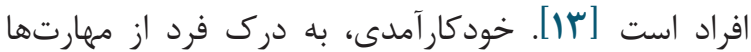

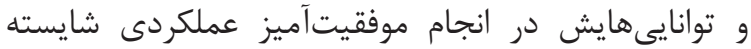
تأكيد دارد. اين مفهوم ميزان تـلاش و و سطح عملكرد فرد فرد

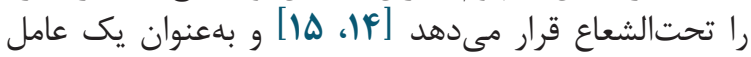

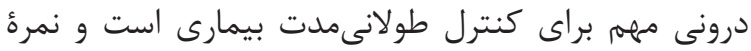

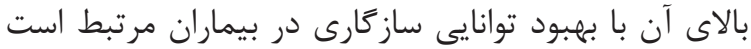

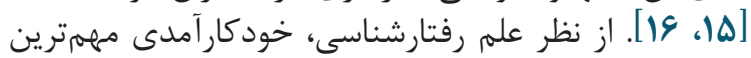

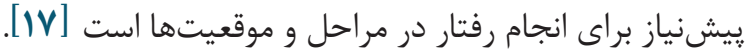

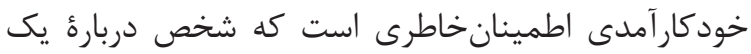

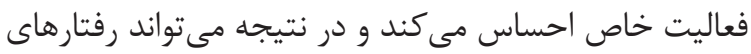

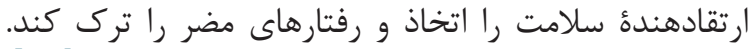

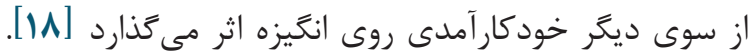

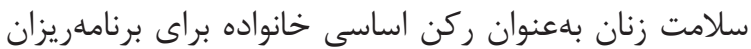

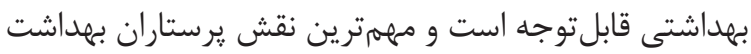

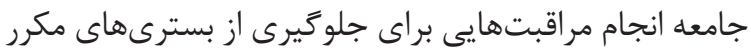

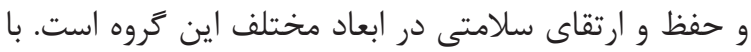

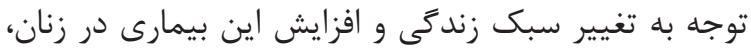

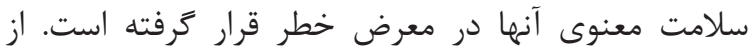
آنجايى كه سلامت معنوى در تطابق و مقابله با اين بيمارى درى

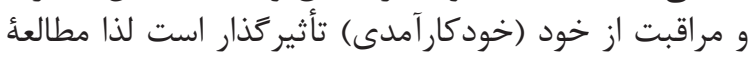

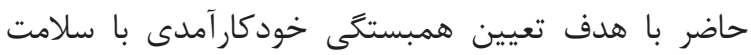
معنوى زنان مبتلا به سرطان سينأ مراجعه كننده بيه مراكز 


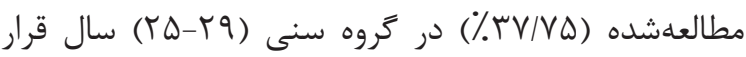

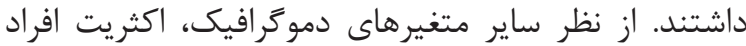

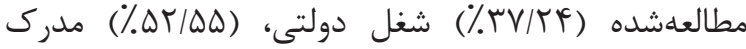

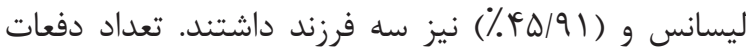

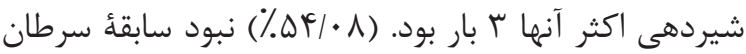

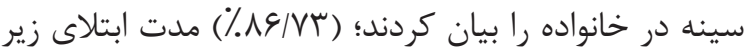

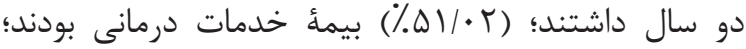

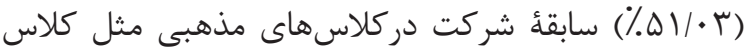

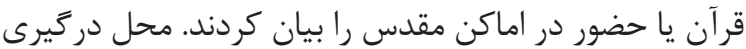

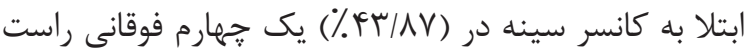

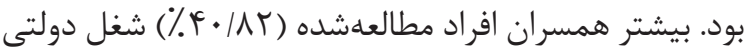

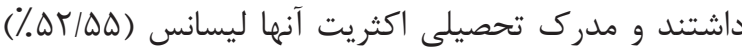

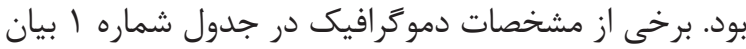

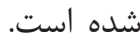

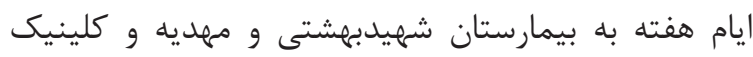

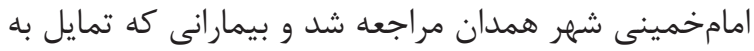

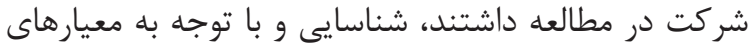

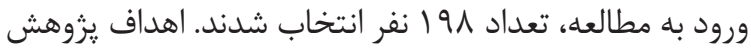

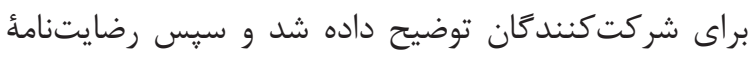

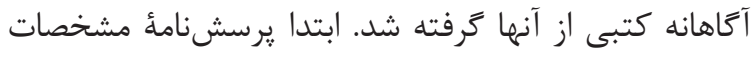

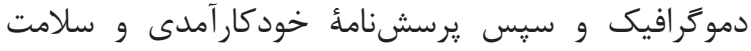

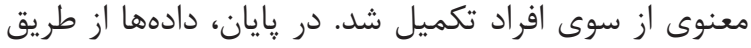

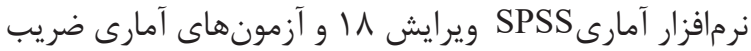

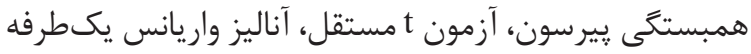

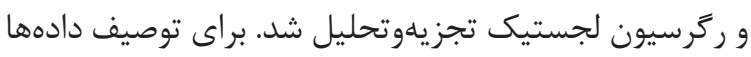
از نسبت، درصد، ميانگين و انحرافتمعيار استفاده شد. شيد.

\section{يافتهها}

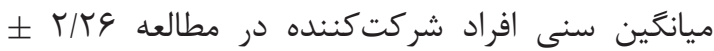

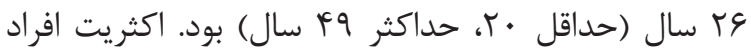

جدول ا. مشخصات دموكَرافيك زنان مبتلا به سرطان سينهُ مر اجعهكننده به مراكز درمانى همدان

\begin{tabular}{|c|c|c|c|}
\hline تعداد (درصد) & 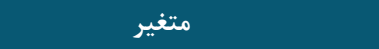 & تعداد (درصد) & متغ \\
\hline \multicolumn{4}{|c|}{ تعداد دفعات شيردهى } \\
\hline$\Delta(T V / \cdot F)$ & 1 & $\Delta G(Y N / \Delta V)$ & $t \cdot-t t^{2}$ \\
\hline$\Delta \cdot(\Gamma \Delta / \Delta I)$ & r & $V f(r V / V \Delta)$ & $r \Delta-r q$ \\
\hline$q \cdot(q \Delta / q))$ & $r$ & $r r(11 / V r)$ & $r \cdot-r q$ \\
\hline \multirow[t]{2}{*}{$\Gamma(1 / \Delta \Gamma)$} & أو و بيشتر & $r V(I T / V V)$ & $r \Delta-r$. \\
\hline & سابقأ سرطان سينه در خانواده & $19(N / 19)$ & بالاى . ب. سال \\
\hline$q \cdot(f \Delta / q 1)$ & 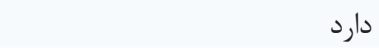 & & شغل \\
\hline \multirow[t]{2}{*}{$1.9(\Delta F / .9)$} & 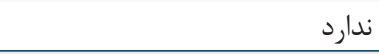 & $V r(T V / T F)$ & دولتى \\
\hline & مدتزمان ابتلا & $\Delta 9(r \cdot / 1 \cdot)$ & قراردادى \\
\hline$I V \cdot(\Lambda \& / V \Psi)$ & زير r سال & $\operatorname{rr}(T r / 94)$ & خانهدار \\
\hline \multirow[t]{2}{*}{$r \varphi(\mid r / T \varphi)$} & r سال و بيشتر & & شغل همسر \\
\hline & سابقهُ شركت در كلاسهاى مذهبى & $\Lambda \cdot(f \cdot / \Lambda r)$ & دولتى \\
\hline $1 \cdot \cdot(\Delta) / \cdot r)$ & دارد & $r \Lambda(\mid F / T \Lambda)$ & قراردادى \\
\hline \multirow[t]{2}{*}{$9 \xi(\uparrow / / q \vee)$} & ندارد & $\Delta r(r \varepsilon \mid \Delta \varphi)$ & آزاد \\
\hline & محل (لوب) دركيرى & 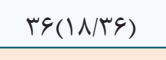 & بيكار \\
\hline$\Lambda \varepsilon(\mathcal{F} / \Lambda \vee)$ & يك جهارم فوقانى راست & & ميزان تحصيلات \\
\hline$F r(Y / / q F)$ & يك جهارم فوقانى خٍ & $9 \cdot(r \cdot \mid q T)$ & زير دييلم \\
\hline$r r(\mid l / V F)$ & يك جهارم تحتانى راست & $r \wedge(\mid F / Y \Lambda)$ & دييلم و فوقدييلم \\
\hline$r \Delta(I T / V Q)$ & يك جهارم تحتانى جِ & $1 \cdot \Gamma(\Delta \Gamma / \Delta \Delta)$ & ليسانس \\
\hline \multirow[t]{6}{*}{$19(9 / 99)$} & مركزى و نوى سينه & $\Delta(\Gamma / \Delta \omega)$ & فوق ليسانس و بالاتر \\
\hline & & & تعداد فرزند \\
\hline & & $9 \cdot(r \cdot|9|)$ & 1 \\
\hline & & $r \wedge(\mid F / Y \Lambda)$ & r \\
\hline & & $1 \cdot r(\Delta \Gamma / \Delta \Delta)$ & r \\
\hline & & $\Delta(\Gamma / \Delta Q)$ & أ و بيشتر \\
\hline
\end{tabular}




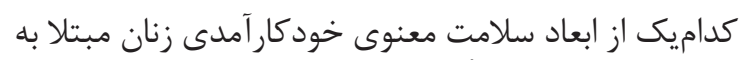

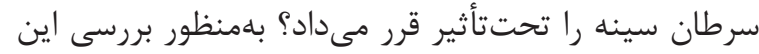

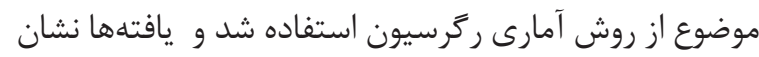

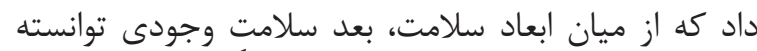
است خودكار آمدى را تحت أثانثير قرار دهد.

\section{بحث}

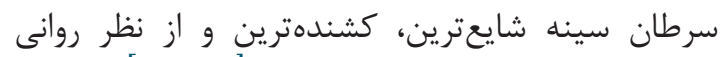

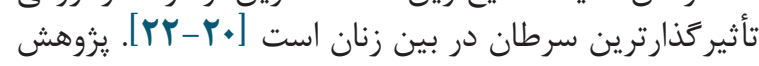

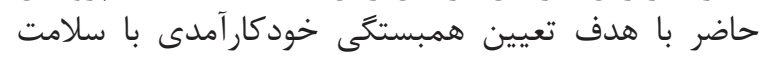

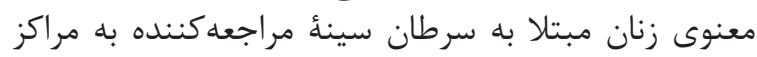

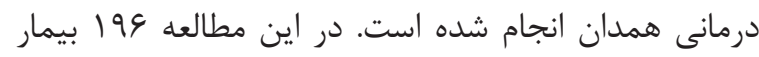

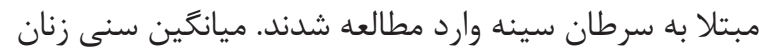

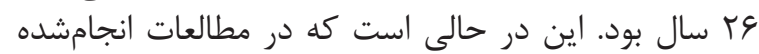

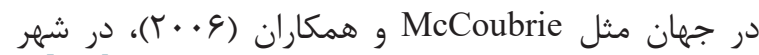

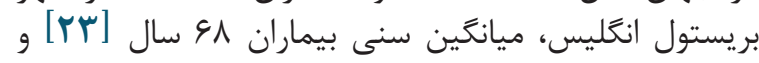

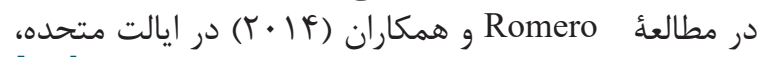

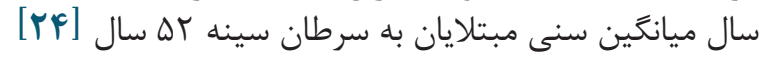

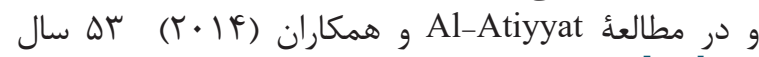

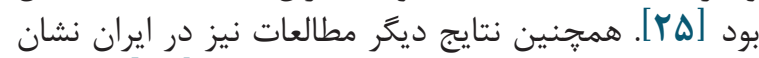

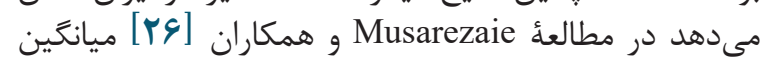

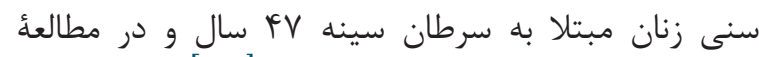

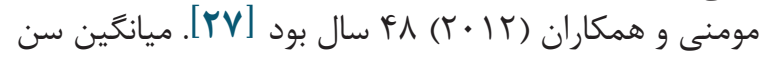

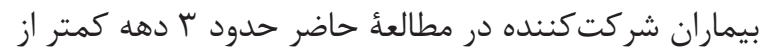

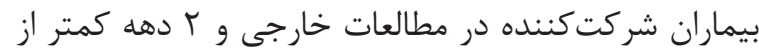

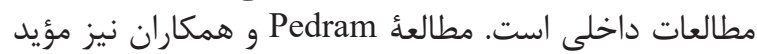

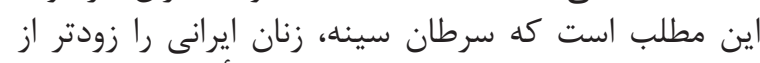

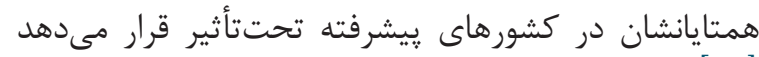

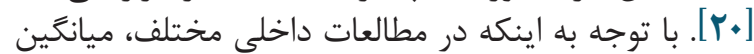

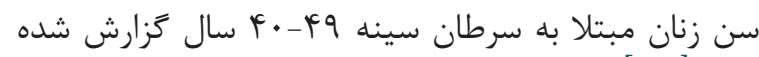

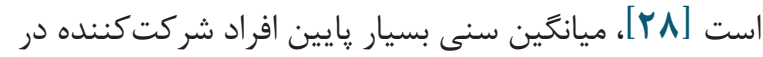

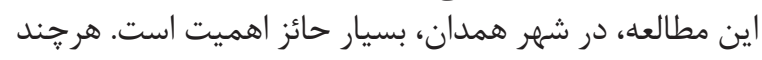

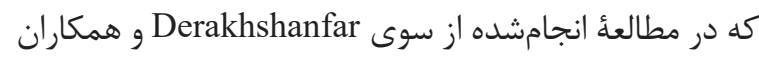

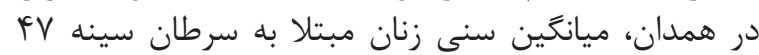

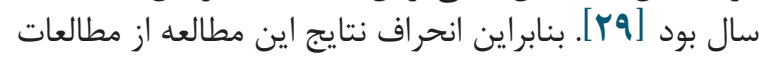

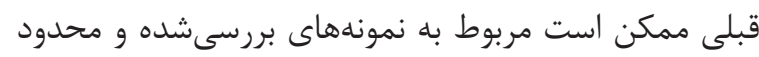

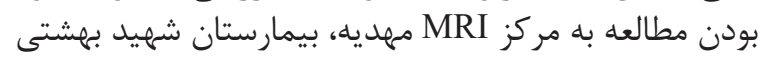

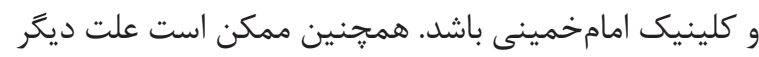

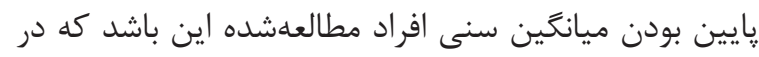

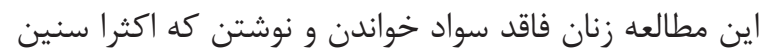
بالايى داشتند، از مطالعه خارج شدند.

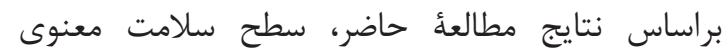

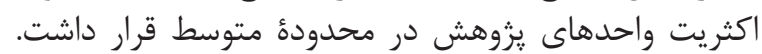

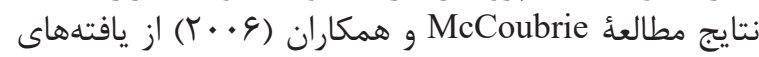

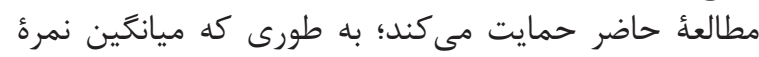

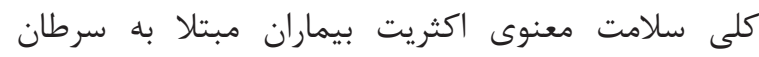

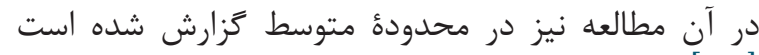

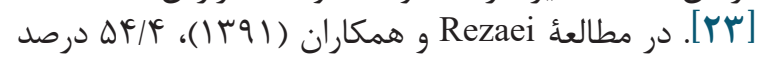

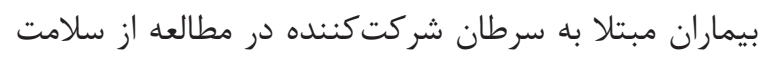

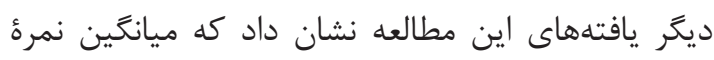
سلامت معنوى

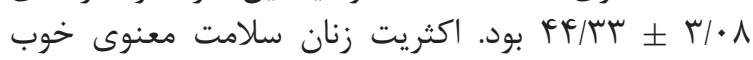

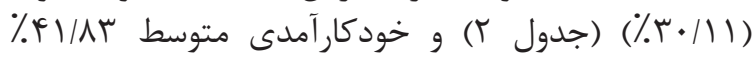

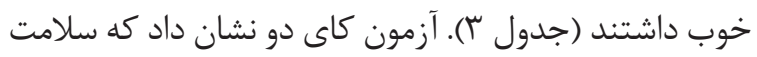

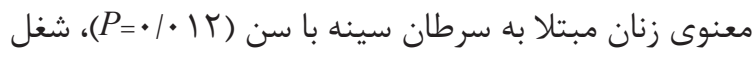
(P)

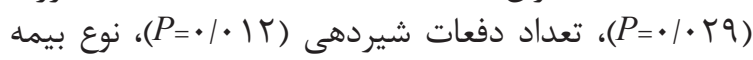

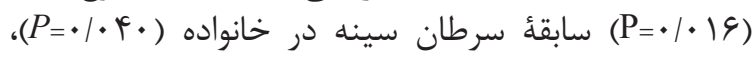

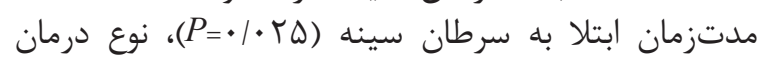

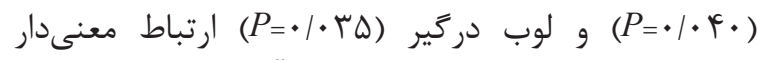

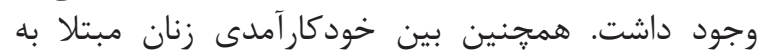

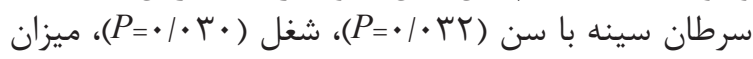

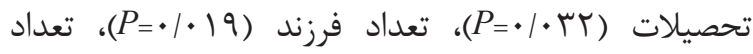

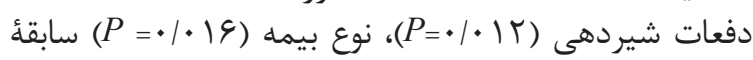

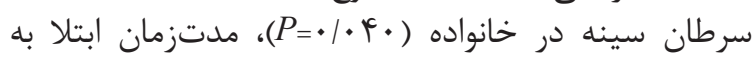

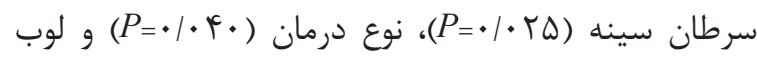

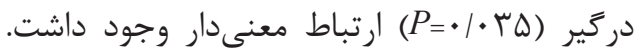

\begin{tabular}{|c|c|c|}
\hline \multicolumn{3}{|c|}{ جدول r. توزيع فراوانى مطلق و نسبى واحدهاى مطالعهشده معندى } \\
\hline درصد & 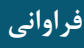 & سلامت معنوى \\
\hline$r \varepsilon 1 . r$ & DI & ضعيف (+F) (Y+F) \\
\hline Fr/AV & 19 & متوسط (9)-99) \\
\hline$r \cdot / 1$ & VT & خوب (•r|-••) \\
\hline $1 \cdots$ & 199 & جمع كل \\
\hline \multicolumn{3}{|c|}{ جدول r. توزيع فراوانى مطلق و نسبى واحد خدهاى مطالعهشده } \\
\hline درصد & فراوانى & خودكار آمدى \\
\hline$r T / 90$ & $i \Delta$ & ضعيف (IV-MF) \\
\hline FI/Ar & NT & متوسط (Iاه-ه r) \\
\hline$r V / . G$ & $\Delta r$ & خوب (AY-GA) \\
\hline N/19 & 19 & 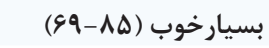 \\
\hline 199 & $1 \ldots$ & جمع كل \\
\hline
\end{tabular}

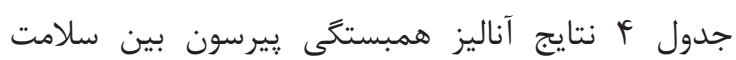

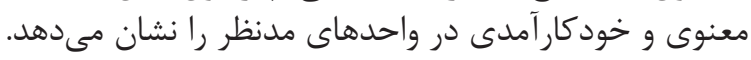

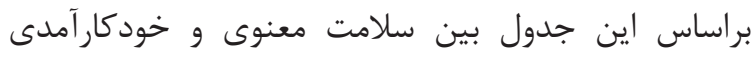

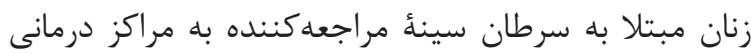

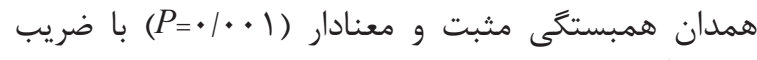

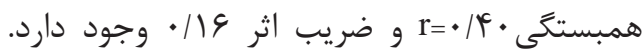

جدول F. همبستكى بين سلامت معنوى و خودكار آمدى در زنان

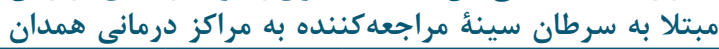

\begin{tabular}{|c|c|c|}
\hline \multicolumn{2}{|c|}{ ضر يب همبستكى بيرسون } & متغيرها \\
\hline sig & $\mathrm{r}$ & سلامت معنوى \\
\hline.. .1 &.$/ 4$. & خودكار آمدى \\
\hline
\end{tabular}


عزتنفس را يُشبينى مى كند، همخوانى دارد [^r]]. مطالعات متعددى در اين زمينه انجام شده است كه

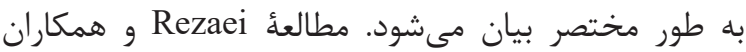

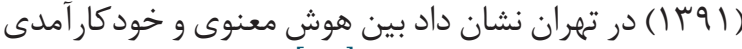

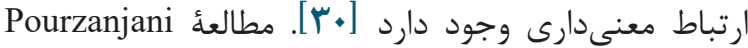

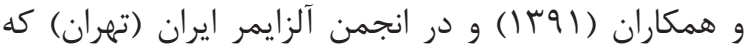

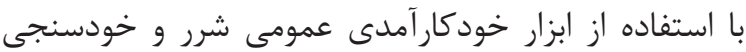

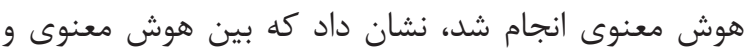

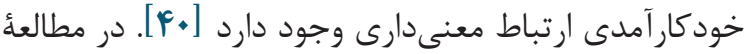
و همكاران (Yller

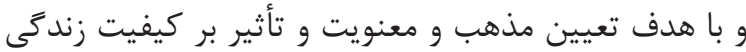

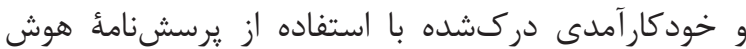

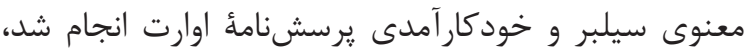

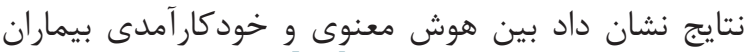

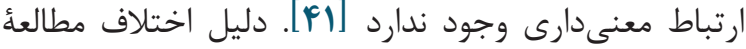

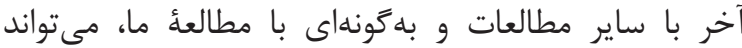

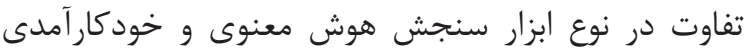

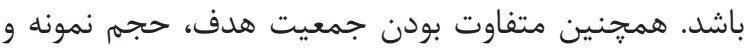

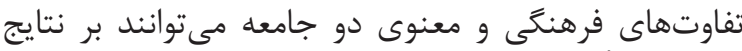

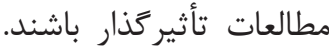

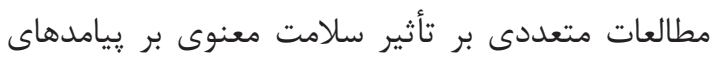

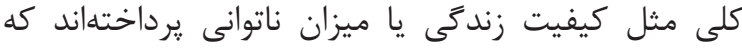

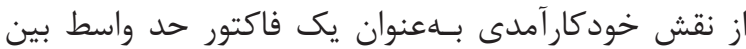

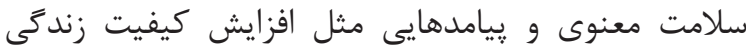

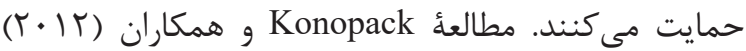

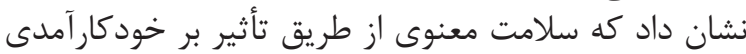

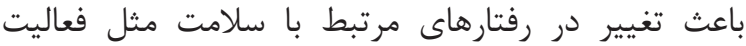

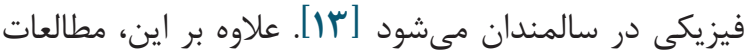

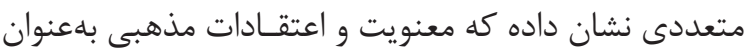

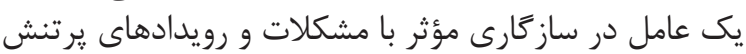

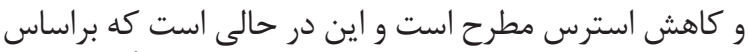

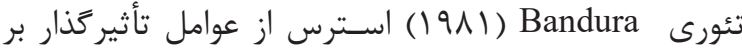

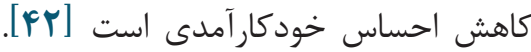

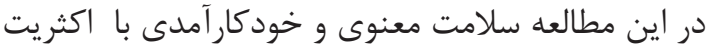

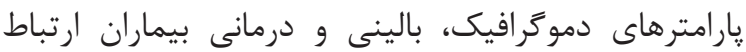

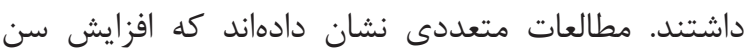

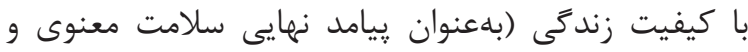

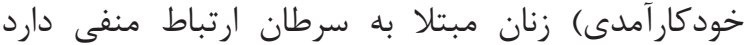

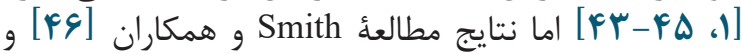

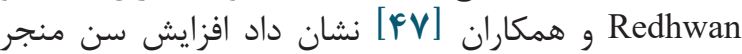

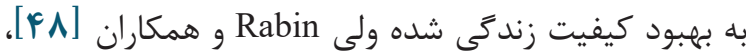
Karami اين زمينه دست نيافتند. شايد علت اين اختلاف دران در نتايج،

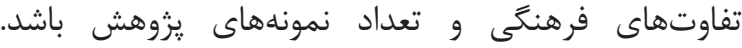

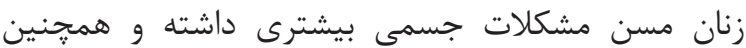

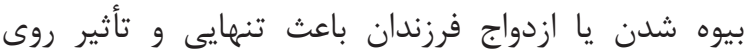

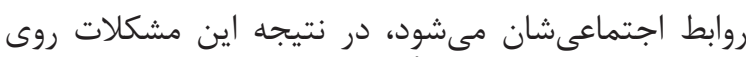

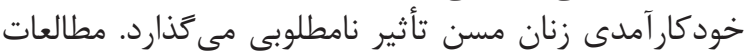

دوره צY شماره Y - خرداد و تير VY

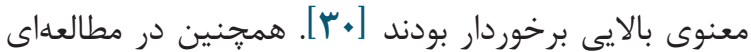

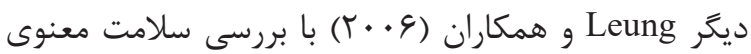

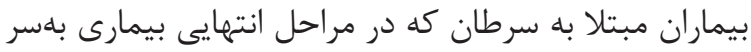

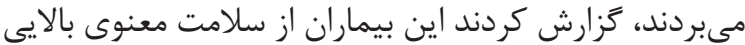

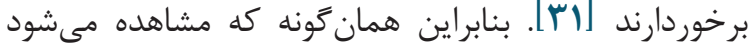

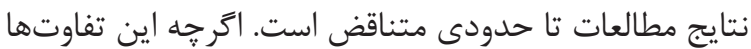

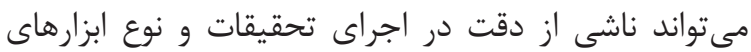

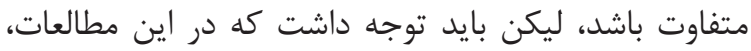

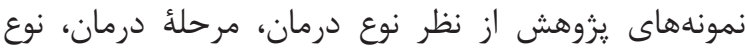

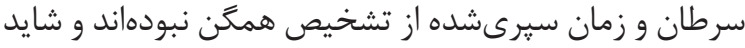
دليل اصلى اين تناقض است.

همجنين در اين مطالعه اكثريت واحدهاى يزوهش

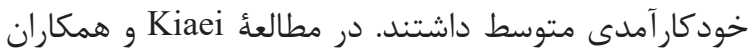

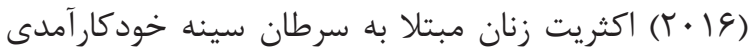

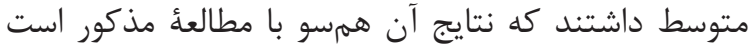

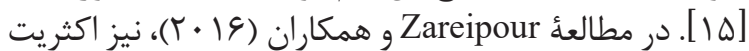

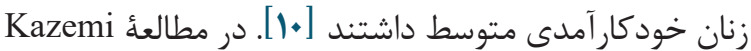

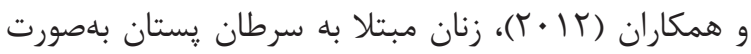

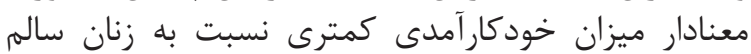

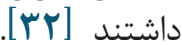

از آنجايى كه در مطالعُّ حاضر، اكثريت افراد سلامت معنوى متوسط و خودكار آمدى متوسط دالئ داشتهانداند، ناخودآكاه

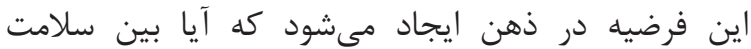

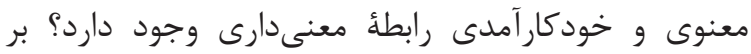

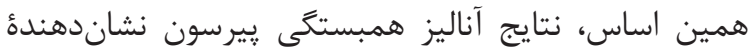

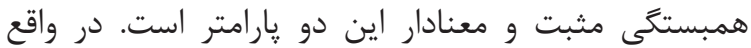

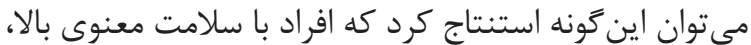

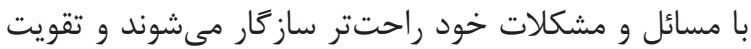

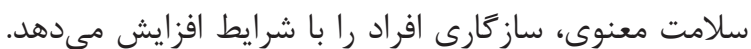

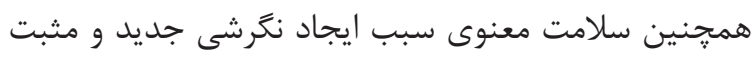

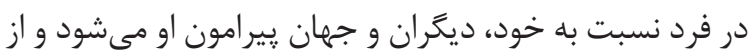

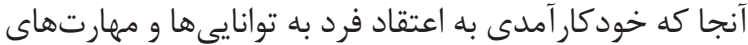

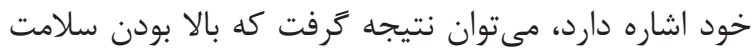

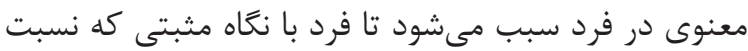

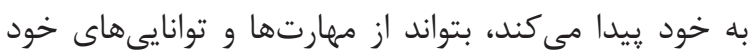

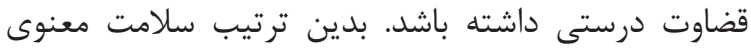

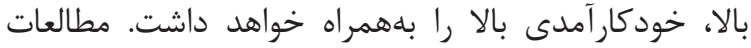

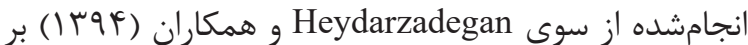

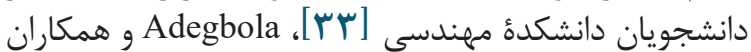

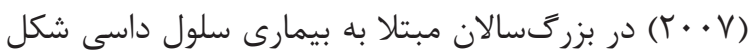

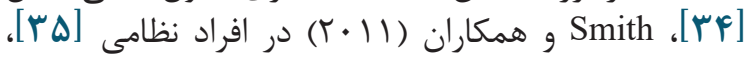
Oareipour

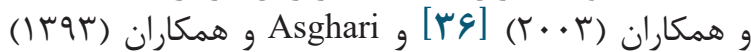

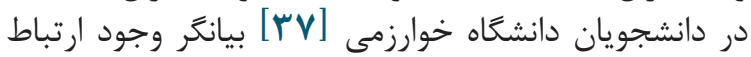

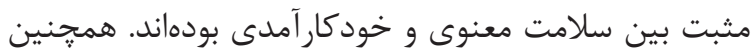

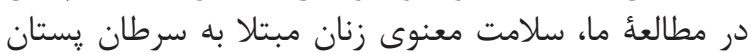

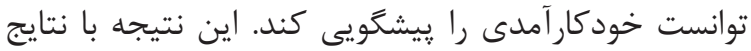

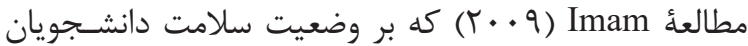
انجام شده بود و نشان داد سلامت معنوى، خودكارآمدى و وآنان

مجله علمى دانشكده يرستارى و مامايى همدان 
يوشش بيمهاى مطلوب بيماران موجب سهولت دسترسى آنها

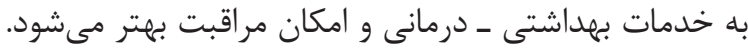

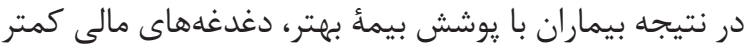

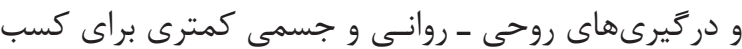

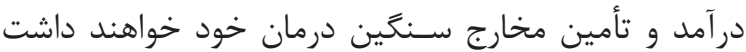

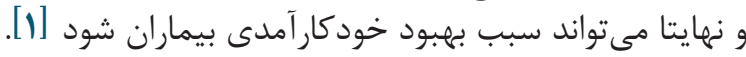
در اين مطالعه بين سلامت معنوى و سابقهُ شركت در مر مارئ

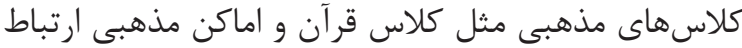

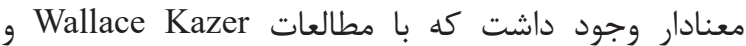

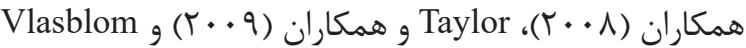

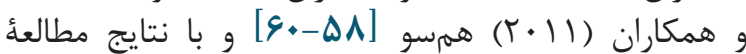
Rahimi

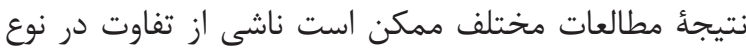
دورة آموزشى و يا كلاس مذهبى مدنظر منات باشد.

\section{نتيجه}

از آنجايى كه در اين مطالعه سلامت معنوى توانايى

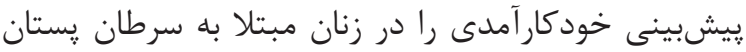

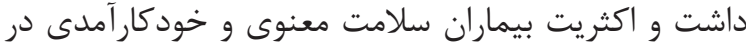

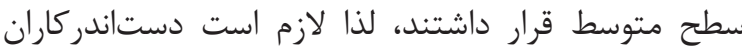

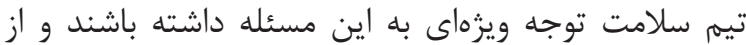

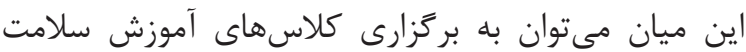

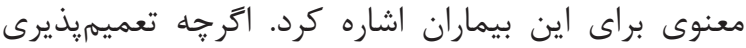

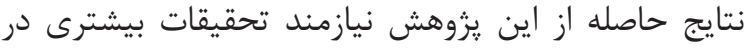

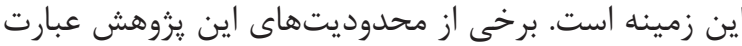

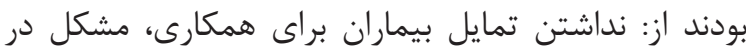

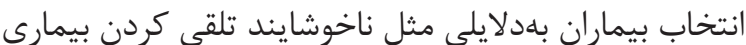

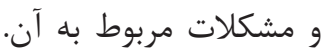

\section{سياسگزارى}

اين مقاله بركرفته از ياياننامهٔ دورئ كارشناسى ارشد

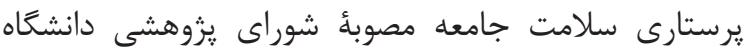

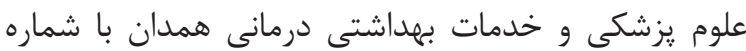

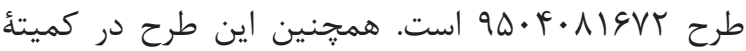

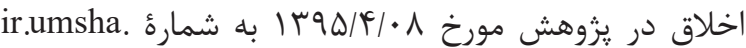
مو 9.rec

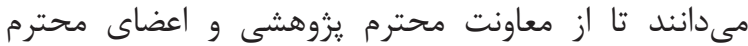

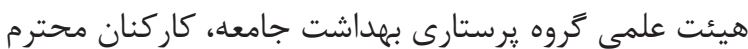

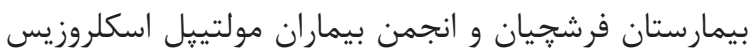

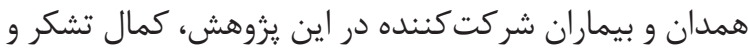
قدردانى را داشته باشند.

\section{تعارض منافع}

در اين مقاله هيجَّونه تعارض منافعى از سوى نويسندكان
بيانكر وجود ارتباط مثبت بين سطح تحصيلات و كيفيت

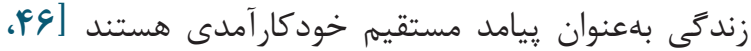

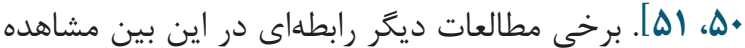

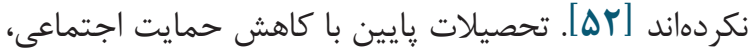

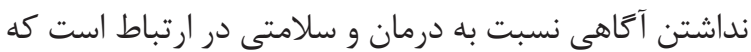

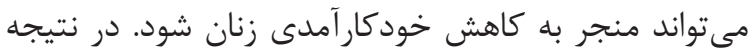

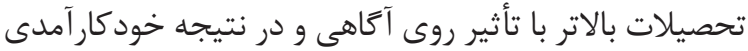

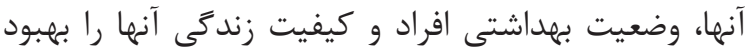

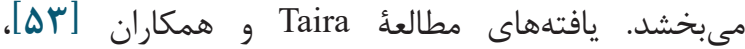

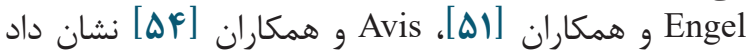

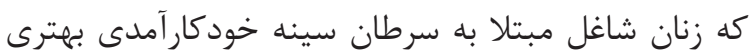

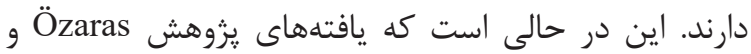

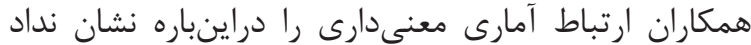

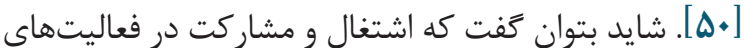

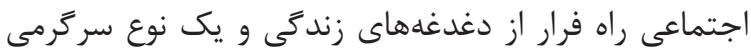

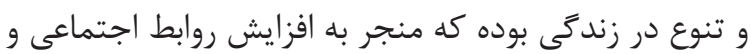

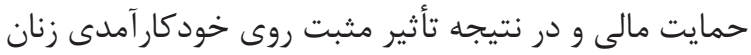

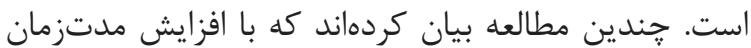

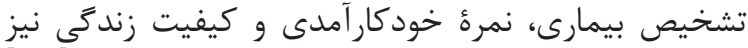

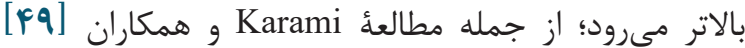

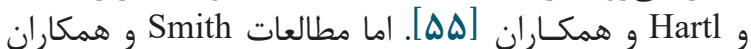

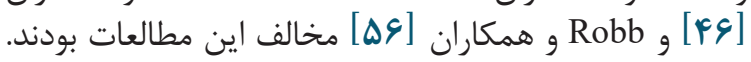

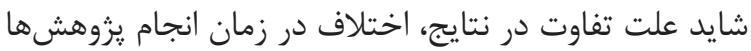

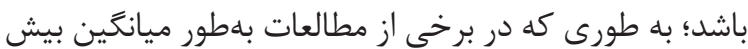

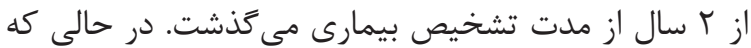

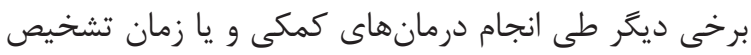

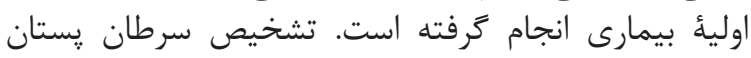

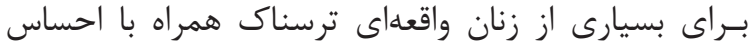

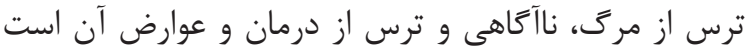

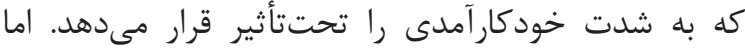

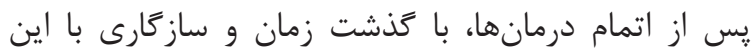

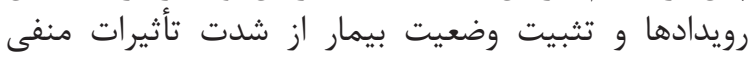
روى خودكارآمدى كاسته مىشود. مطالعات

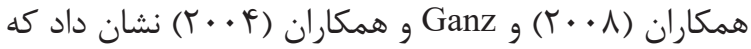

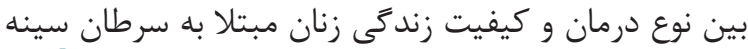

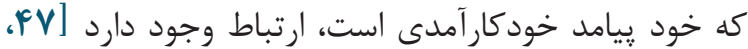

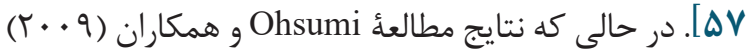

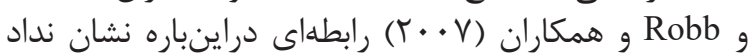

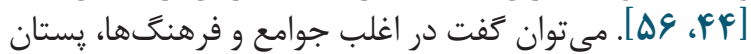

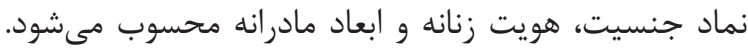

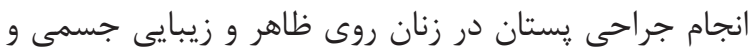

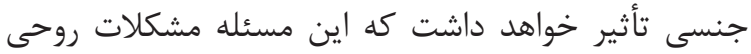

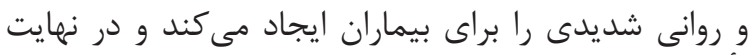

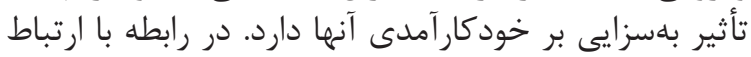

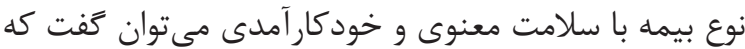




\section{References}

1. Monfared A, Pakseresht S, Ghanbari Khanghah A, Atrkar-Roshan Z. Health-Related Quality of Life and its related factors among women with breast cancer. J Holist Nurs Midwifery. 2013;23(2):52-62.

2. Momeni T, Musarezaie A, Karimian J, Ebrahimi A. The relationship between spiritual well-being and depression in Iranian breast cancer patients. Health Inf Manage. 2012;8(8):1007-16.

3. Mardani Hamule M, Shahraky Vahed A. The Assessment of Relationship between Mental Health and Quality of Life in Cancer Patients. Avicenna Journal of Clinical Medicine. 2009;16(2):33-8.

4. Matsushita T, Matsushima E, Maruyama M. Psychological state, quality of life, and coping style in patients with digestive cancer. Gen Hosp Psychiatry. 2005;27(2):125-32. http://psycnet.apa. org/doi/10.1016/j.genhosppsych.2004.10.006 PMID: 15763124

5. Bekelman DB, Dy SM, Becker DM, Wittstein IS, Hendricks DE, Yamashita TE, et al. Spiritual well-being and depression in patients with heart failure. J Gen Intern Med. 2007;22(4):470-7. https://dx.doi.org/10.1007\% 2Fs11606-006-0044-9 PMID:17372795 PMCID:PMC182942 1

6. Kamian S, Taghdisi MH, Azam K, Estebsari F. Evaluating Effectiveness of Spiritual Health Education on Increasing Hope among Breast Cancer Patients. Iran J Health Educ Health Promot. 2014;2(3):208-14. http://journal.ihepsa.ir/article-1-193-en.html

7. Shahsavari A, Mahmodi M, Hayati F. Relationship to subjective well being and demographic variables in women with breast cancer refered to hospitals affiliated to medical sciences universities of Tehran city, 1386. Iran J Breast Dis. 2009;2(1):23-8. $\quad$ http://ijbd.ir/article-1-70-fa. html

8. Mohamad Karimi M, Shariatnia K. Effectiveness of spiritual therapy on the life quality of the women with breast cancer in tehran. J Urmia Nurs Midwifery Fac. 2017;15(2):107-18. http:// unmf.umsu.ac.ir/article-1-3064-en.html

9. Herrman H, Saxena S, Moodie R, World Health Organization. Promoting mental health: concepts, emerging evidence, practice: a report of the World Health Organization, Department of Mental Health and Substance Abuse in collaboration with the Victorian Health Promotion Foundation and the University of Melbourne: World Health Organization; 2005. http://www.who.int/mental health/evidence/en/promoting mhh.pdf

10. Zareipour M, Abdolkarimi M, Asadpour M, Dashti S, Askari F. The Relationship between Spiritual
Health and Self-efficacy in Pregnant Women Referred to Rural Health Centers of Uremia in 2015. Community Health Journal. 2016;10(2):52-61. http://chj.rums.ac.ir/article 45787 en.html

11. Allahbakhshian M, Jaffarpour M, Parvizy S, Haghani H. A Survey on relationship between spiritual wellbeing and quality of life in multiple sclerosis patients. Zahedan J Res Med Sci. 2010;12(3):29-33. http://zjrms.ir/article-1-676en.html

12. Jesse DE, Reed PG. Effects of spirituality and psychosocial well-being on health risk behaviors in Appalachian pregnant women. J Obstet Gynecol Neonatal Nurs. 2004;33(6):739-47. https://doi.org/10.1177/0884217504270669 PMID: 15561662

13. Konopack JF, McAuley E. Efficacy-mediated effects of spirituality and physical activity on quality of life: A path analysis. Health Qual Life Outcomes. 2012;10(1):57. https:// dx.doi.org/10.1186\%2F 1477-7525-10-57 PMID:22642832 PMCID:PMC3406955

14. Frei A, Svarin A, Steurer-Stey C, Puhan MA. Self-efficacy instruments for patients with chronic diseases suffer from methodological limitations-a systematic review. Health Qual Life Outcomes. 2009;7(1):86. https:// dx.doi.org/10.1186\%2F 1477-7525-7-86 PMID:19781095 PMCID:PMC2761851

15. Kiaei M, Ferdosi M, Moradi R, Chelongar Q, Ahmadzadeh M, Bahmanziari N. Association between self-efficacy and quality of life in women with breast cancer undergoing chemotherapy. J Qazvin Univ Med Sci. 2016;20(2):58-65.

16. Salimian SH, Esmaieli R, Dabirian A, Mansoorin $\mathrm{S}$, Zohari Anboohi S. The survey of factors related to self-efficacy based on Orem's theory in patients with coronary artery bypass surgery in Tehran in 2014. Advances in Nursing \& Midwifery. 2016;25(90):9-16.

17. Bandura A. Self-efficacy: toward a unifying theory of behavioral change. Psychol Rev. 1977;84(2):191-215. PMID:847061

18. Tam C-L, Chong A, Kadirvelu A, Khoo Y-T. Parenting styles and self-efficacy of adolescents: Malaysian scenario. Global Journal of Human-Social Science Research. 2013;12(14-A).

19. Barati S. Examine the simple and multiple relationship of self-efficacy, self-esteem, self-discovery and academic performance of the new system third year students in Ahvaz [dissertation]. Ahvaz Iran, Shahid Chamran University of Ahvaz; 1997.

20. Pedram M, Mohammadi M, Naziri G, Aeinparast 
N. Effectiveness of cognitive-behavioral group therapy on the treatment of anxiety and depression disorders and on raising hope in women with breast cancer. Woman and Society. 2011;1(4):3461. http://jzvj.miau.ac.ir/article 1217 en.html

21. Mehrinejad S, Shariati S, Shapouri M. Stress in Women with Breast Cancer Compared to the Healthy Ones. Clinical Psychology \& Personality. 2010;1(43):49-56. http://cpap.shahed.ac.ir/ article-1-503-en.html

22. Sajadi HM, Salehi B, Moshfeghi K. Comparison effect of drug therapy and drug-cognitive therapy on decreasing depression in women with breast cancer. J Arak Uni Med Sci . 2008;11(3):51-5. http://amuj.arakmu.ac.ir/article-1-203-en.html

23. McCoubrie RC, Davies AN. Is there a correlation between spirituality and anxiety and depression in patients with advanced cancer? Support Care Cancer. 2006;14(4):379-85. https://doi.org/10.1007/ s00520-005-0892-6 PMID: $\underline{16283208}$

24. Romero C, Friedman LC, Kalidas M, Elledge R, Chang J, Liscum KR. Self-forgiveness, spirituality, and psychological adjustment in women with breast cancer. J Behav Med. 2006;29(1):2936. https://doi.org/10.1007/s10865-005-9038-z PMID: 16362244

25. Al-Atiyyat NM, Naga BSB. Effects of Spirituality in Breast Cancer Survivors: Critique of Quantitative Research. World Fam Med J. 2014;12(4):2533. https://doi.org/10.5742/MEFM.2014.92466

26. Musarezaie A, Momeni Ghaleghasemi T, Ebrahimi A, Karimian J. The Relationship between Spiritual Wellbeing with Stress, Anxiety, and Some Demographic Variables in Women with Breast Cancer Referring to the Specialized Cancer Treatment Center in Isfahan. Iran J Health Syst Res. 2012;8(1):104-13.

27. Jadidi A, Farahaninia M, Janmohammadi S, Haghani H. The Relationship between Spiritual Well-Being and Quality of Life among Elderly People Residing in Kahrizak Senior House. Iran J Nurs. 2011;24(72):48-56. http://ijn.iums.ac.ir/ article-1-1025-en.html

28. Harirchi I, Karbakhsh M, Kashefi A, Momtahen AJ. Breast cancer in Iran: results of a multi-center study. Asian Pac J Cancer Prev. 2004;5(1):24-7. PMID: 15075000

29. Derakhshanfar A, Niayesh A, Abbasi M, Ghalaeeha A, Shojaee M. Frequency of depression in breast cancer patients: a study in farshchian and besat hospitals of hamedan during 2007-8. Iran J Surg. 2013;21(2):68-74. http://www.sid.ir/En/ Journal/ViewPaper.aspx?ID=493553

30. Rezaei M, Seyedfatemi N, Hosseini F. Spiritual Well-being in Cancer Patients who Undergo Chemotherapy. Hayat. 2008;14(4 and 3):33-9.

$$
\text { • 1. بررسى همبستخى خود كار آمدى با ... }
$$

http:/hayat.tums.ac.ir/article-1-135-en.html

31. Leung K-K, Chiu T-Y, Chen C-Y. The influence of awareness of terminal condition on spiritual well-being in terminal cancer patients. J Pain Symptom Manage. 2006;31(5):449-56. https:// doi.org/10.1016/j.jpainsymman.2006.02.001 PMID: 16716875

32. Kazemi M, Momeni M. Comparative evaluation of self-efficacy and empowerment in women with breast cancer and healthy women. Iran J Breast Dis. 2012;5(1):45-53.

33. Heydarzadegan A, Kochakzaei M. Study of the relationship between spiritual well-being and self-efficacy of students of faculty of engineering and psychology and educational sciences. Majallah-i Amuzih-i Muhandisi-i Iran. 2015;17(65):79.

34. Adegbola MA. The Relationship among spirituality, self-efficacy, and quality of life in adults with sickle cell disease. Texas: The university of Texas at Arlington; 2007.

35. Smith II JP. The effects of self-efficacy and spirituality on the job satisfaction and motivation to lead among redeploying soldiers as moderated by transformational leadership. Virginia: Regent University; 2011.

36. Oman D, Hedberg J, Downs D, Parsons D. A transcultural spiritually based program to enhance caregiving self-efficacy: A pilot study. Complementary Health Practice Review. 2003;8(3):20124. https://doi.org/10.1177/1076167503250796

37. Asghari F, Saadat S, Atefi Karajvandani S, Janalizadeh Kokaneh S. The Relationship between Academic Self-Efficacy and Psychological Well-Being, Family Cohesion, and Spiritual Health among Students of Kharazmi University. Iran J Med Educ. 2014;14(7):581-93. http:// ijme.mui.ac.ir/article-1-3250-en.html

38. Imam SS, Nurullah AS, Makol-Abdul PR, Rahman SA, Noon HM. Spiritual and psychological health of Malaysian youths. Vol 20. Research in the Social Scientific Study of Religion: Brill; 2009. p. $85-102$.

39. Esmaeili PZS. Assessment of correlation between self-efficacy and spiritual intelligence of family caregivers of elderly patient with alzheimer in tehran 1391 [dissertation]. Tehran Iran: Islamic Azad University Medical Sciences of Tehran; 2016.

40. Esmaeili PZS, Mashouf S, Safari Z, Abbasi M. The relationship between self-efficacy and spiritual intelligence among carers of a family member of the elderly with Alzheimer's disease. Medical Figh Quarterly. 2012;4(11-12):151-66.

41. Miller JF, McConnell TR, Klinger TA. Religiosity and spirituality: Influence on quality of life and perceived patient self-efficacy among cardi- 
ac patients and their spouses. Journal of Religion and Health. 2007;46(2):299-313. https://doi. org/10.1007/s10943-006-9070-5

42. Bandura A, Schunk DH. Cultivating competence, self-efficacy, and intrinsic interest through proximal self-motivation. J Pers Soc Psychol. 1981;41(3):586. https://doi.org/10.1037/0022$\underline{3514.41 .3 .586}$

43. Härtl K, Engel J, Herschbach P, Reinecker H, Sommer H, Friese K. Personality traits and psychosocial stress: quality of life over 2 years following breast cancer diagnosis and psychological impact factors. Psychooncology. 2010;19(2):160-9. https://doi.org/10.1002/ pon.1536 PMID:19189279

44. Ohsumi S, Shimozuma K, Morita S, Hara F, Takabatake D, Takashima S, et al. Factors associated with health-related quality-of-life in breast cancer survivors: influence of the type of surgery. Jpn J Clin Oncol. 2009 39(8):491-6. https://doi. org/10.1093/ijco/hyp060 PMID:19520689

45. Ganz PA, Guadagnoli E, Landrum MB, Lash TL, Rakowski W, Silliman RA. Breast cancer in older women: quality of life and psychosocial adjustment in the 15 months after diagnosis. J Clin Oncol. 2003;21(21):4027-33. https://doi. org/10.1200/JCO.2003.08.097 PMID: 14581426

46. Smith AW, Alfano CM, Reeve BB, Irwin ML, Bernstein L, Baumgartner K, et al. Race/ethnicity, physical activity, and quality of life in breast cancer survivors. Cancer Epidemiol Biomarkers Prev. 2009;18(2):656-63. https://dx. doi.org/10.1158\%2F1055-9965.EPI-08-0352 PMID:19190157 PMCID:PMC3031117

47. Redhwan A, Idris MM, Zaleha M, Robert C, Fuad I, Sami R. Quality of life among women with breast cancer from Universiti Kebangsaan Malaysia Medical Centre, Malaysia. J Community Health. 2008;14(2):46-55.

48. Rabin EG, Heldt E, Hirakata VN, Fleck MP. Quality of life predictors in breast cancer women. Eur J Oncol Nurs. 2008;12(1):537. https://doi.org/10.1016/j.ejon.2007.06.003 PMID:17884731

49. Karami O, Falahat-Pisheh F, Jahani H, Beiraghdar N. Quality of life in cancer patients in Qazvin 2007. J Qazvin Univ Med Sci. 2010;14(3):80-7.

50. Özaras G, Özyurda F. Quality of life and influencing factors in patients with a gynaecologic cancer diagnosis at Gazi University, Turkey. Asian Pac J Cancer Prev. 2010;11(5):1403-8. PMID:21198301

51. Engel J, Kerr J, Schlesinger-Raab A, Eckel R, Sauer H, Hölzel D. Predictors of quality of life of breast cancer patients. Acta Oncologica. 2003;42(7):710-8. PMID:14690156
52. Kwan ML, Ergas IJ, Somkin CP, Quesenberry CP, Neugut AI, Hershman DL, et al. Quality of life among women recently diagnosed with invasive breast cancer: the Pathways Study. Breast Cancer Res Treat. 2010;123(2):507-24. https:// dx.doi.org/10.1007\%2Fs 10549-010-0764-8 PMID:20140494 PMCID:PMC2935682

53. Taira N, Shimozuma K, Shiroiwa T, Ohsumi S, Kuroi K, Saji S, et al. Associations among baseline variables, treatment-related factors and health-related quality of life 2 years after breast cancer surgery. Breast Cancer Res Treat. 2011;128(3):735-47. $\quad$ https://doi.org/10.1007/ s10549-011-1631-y $\quad$ PMID:21681445

54. Avis NE, Crawford S, Manuel J. Quality of life among younger women with breast cancer. J Clin Oncol. 2005;23(15):3322-30. https://doi. org/10.1200/JCO.2005.05.130 PMID: $\underline{15908646}$

55. Härtl K, Janni W, Kästner R, Sommer H, Strobl B, Rack B, et al. Impact of medical and demographic factors on long-term quality of life and body image of breast cancer patients. Ann Oncol. 2003;14(7):1064-71. PMID:12853348

56. Robb C, Haley WE, Balducci L, Extermann M, Perkins EA, Small BJ, et al. Impact of breast cancer survivorship on quality of life in older women. Crit Rev Oncol Hematol. 2007;62(1):84-91. https://doi.org/10.1016/j.critrevonc.2006.11.003 PMID: 17188505

57. Ganz PA, Kwan L, Stanton AL, Krupnick JL, Rowland JH, Meyerowitz BE, et al. Quality of life at the end of primary treatment of breast cancer: first results from the moving beyond cancer randomized trial. J Natl Cancer Inst. 2004;96(5):376-87. PMID: 14996859

58. Wallace M, Campbell S, Grossman SC, Shea JM, Lange JW, Quell TT. Integrating spirituality into undergraduate nursing curricula. Int J Nurs Educ Scholarsh. 2008;5(1):1-13. $\quad$ https://doi. org/10.2202/1548-923X.1443 PMID: 18384268

59. Taylor EJ, Mamier I, Bahjri K, Anton T, Petersen F. Efficacy of a self-study programme to teach spiritual care. J Clin Nurs. 2009;18(8):1131-40. https://doi.org/10.1111/ j.1365-2702.2008.02526.x PMID: 19207790

60. Vlasblom JP, van der Steen JT, Knol DL, Jochemsen H. Effects of a spiritual care training for nurses. Nurse Educ Today. 2011;31(8):7906. https://doi.org/10.1016/j.nedt.2010.11.010 PMID:21146259

61. Rahimi N, Nouhi E, Nakhaee N. Spiritual Well-being and Attitude toward Spirituality and Spiritual Care in Nursing and Midwifery Students. Iran J Nurs. 2013;26(85):55-65. http://ijn. iums.ac.ir/article-1-1690-en.html 